\title{
Editorial: Exploring Novel Approaches to Eliminate HIV Reservoirs to Achieve a Cure for HIV
}

\author{
Renée M. van der Sluis ${ }^{1,2 *}$, Andrés Finzi ${ }^{3,4,5}$ and Matthew S. Parsons ${ }^{6,7 *}$ \\ ${ }^{1}$ Aarhus Institute of Advanced Studies (AIAS), Aarhus University, Aarhus, Denmark, ${ }^{2}$ Department of Biomedicine, Aarhus \\ University, Aarhus, Denmark, ${ }^{3}$ Département de Microbiologie, Infectiologie et Immunologie, Université de Montréal, Montreal, \\ QC, Canada, ${ }^{4}$ Department of Microbiology and Immunology, McGill University, Montreal, QC, Canada, ${ }^{5}$ Centre de \\ Recherche du CHUM, Montreal, QC, Canada, ${ }^{6}$ Yerkes National Primate Research Center, Emory University, Atlanta, GA, \\ United States, ${ }^{7}$ Department of Pathology and Laboratory Medicine, School of Medicine, Emory University, Atlanta, GA, \\ United States
}

Keywords: human immunodeficiency virus (HIV), HIV cure, HIV cure research, HIV cure strategies, HIV latency

Editorial on the Research Topic

Exploring Novel Approaches to Eliminate HIV Reservoirs to Achieve a Cure for HIV

\section{OPEN ACCESS}

Edited and reviewed by:

Colleen B. Jonsson,

University of Tennessee Health

Science Center (UTHSC),

United States

*Correspondence:

Renée M. van der Sluis renee@biomed.au.dk

Matthew S. Parsons

matthew.s.parsons@emory.edu

Specialty section: This article was submitted to Virus and Host, a section of the journal

Frontiers in Cellular and

Infection Microbiology

Received: 26 January 2021 Accepted: 02 February 2021 Published: 25 February 2021

Citation:

van der Sluis RM, Finzi $A$ and Parsons MS (2021) Editorial: Exploring Novel Approaches to Eliminate HIV Reservoirs to Achieve a Cure for HIV. Front. Cell. Infect. Microbiol. 11:658848. doi: 10.3389/fcimb.2021.658848
Antiretroviral therapy (ART) has revolutionized the treatment of HIV and has dramatically reduced morbidity and mortality among people living with HIV (PLWH) (Antiretroviral Therapy Cohort Collaboration, 2017). ART inhibits HIV replication, decreasing viral loads, halting disease progression and preventing sexual transmission of the virus (Attia et al., 2009; Volberding and Deeks, 2010; Cohen et al., 2011; Bavinton et al., 2018; Rodger et al., 2019). Cessation of ART, however, allows HIV to re-initiate replication (Davey et al., 1999; Colby et al., 2018). As such, ART is required throughout the lifespan of PLWH. Treatments that aim to cure HIV or induce viral remission are highly desirable to alleviate the need for lifelong ART.

The primary barrier to curing HIV is the persistence of a latent viral reservoir that predominantly resides in $\mathrm{CD}^{+} \mathrm{T}$ cells (Chun et al., 1997; Finzi et al., 1997; Wong et al., 1997). Numerous strategies have been proposed to target this reservoir (Pitman et al., 2018). Currently, the predominant strategy is the 'shock and kill' approach, which proposes employing latency reversing agents (LRAs) to induce virus expression and make virus harboring cells visible to the immune system. Subsequently, immune cells are engaged to eliminate cells harboring reactivated latent virus. Although clinical trials exploring the utility of the 'shock and kill' strategy have shown LRAs to induce viral expression in ART-treated PLWH, evidence of the efficacy of this approach for decreasing the frequency of cells carrying integrated HIV DNA has yet to be generated (Dufour et al., 2020). The reason(s) that 'shock and kill' strategies have not reduced the frequency of cells carrying integrated HIV DNA remains unclear. Novel LRAs and/or more potent anti-viral immune responses may be required to purge the virus (Kim et al., 2018; Dufour et al., 2020).

In this special issue of Frontiers in Cellular and Infection Microbiology, we invited early stage investigators to contribute their ideas as to how to tackle the important scientific problem of curing HIV infection. We received eight manuscripts, which we will briefly summarize to introduce this special issue.

Thomas et al. provide an excellent overview of recent advances towards understanding the latent viral reservoir and highlight various potential strategies for its eradication. To measure the success of these strategies, highly sensitive and accurate assays are required. Measuring the latent HIV 
reservoir is made difficult by variation in the viral genome, the low frequency of cells carrying latent virus and the presence of defective proviruses. Thomas et al. discuss the different methods that are available and debate the advantages and disadvantages of these assays.

Takahama and Yamamoto review the potential of using ligands for pattern recognition receptors (PRRs) as a cure strategy. PRR ligands can be used as vaccine adjuvants to enhance activation of the innate immune system and promote antigen-specific immune responses. PRR ligands can also be used to enhance preexisting immune responses. PRR ligands fit the 'shock and kill' approach well, because they can be used to activate the latent virus (i.e. deliver the "shock") and boost the anti-viral immune response (i.e. the "kill"). Additionally, Takahama and Yamamoto discuss the possibility of combining PRR ligands with other immunotherapies, such as therapeutic vaccines, checkpoint inhibitors and broadly neutralizing antibodies (bNAbs).

Five of the articles in this special issue provide novel ideas as to how to enhance anti-viral immune responses through the use immunotherapeutic approaches.

Holder and Grant review the potential utility of immune checkpoint blockers targeting TIGIT to invigorate anti-viral immune responses. The advantage of using anti-TIGIT over other immune checkpoint blockers is that TIGIT is expressed on many HIV-specific CD8 ${ }^{+}$T cells in PLWH (Chew et al., 2016; Tauriainen et al., 2017) and blocking TIGIT can reinvigorate exhausted $\mathrm{T}$ cell responses. Additionally, anti-TIGIT therapy may also enhance NK cell function.

$\mathrm{Mu}$ et al. summarize recent developments pertaining to HIVspecific CAR T cells. One of the challenges $\mathrm{Mu}$ et al. describe is that CAR T cells need to function under conditions where low amounts of HIV-antigen are present. They discuss how combining CAR T cell therapy with LRAs might increase the effectiveness of this therapeutic option.

Juno and Kent summarize the different subsets of gammadelta- $(\gamma \delta) \mathrm{T}$ cells and review their immunotherapeutic potential in HIV cure strategies. A major clinical advantage of employing these cells is the lack of MHC restriction for $\gamma \delta$ T cell-mediated killing. This provides an opportunity for an "off-the-shelf" allogeneic product, circumventing the MHC-restriction present for most other cell-based immunotherapies.

\section{REFERENCES}

Antiretroviral Therapy Cohort Collaboration (2017). Survival of HIV-positive patients starting antiretroviral therapy between 1996 and 2013: a collaborative analysis of cohort studies. Lancet HIV 4, e349-e356. doi: 10.1016/S2352-3018 (17)30066-8

Attia, S., Egger, M., Muller, M., Zwahlen, M., and Low, N. (2009). Sexual transmission of HIV according to viral load and antiretroviral therapy: systematic review and meta-analysis. AIDS 23, 1397-1404. doi: 10.1097/ QAD.0b013e32832b7dca

Bavinton, B. R., Pinto, A. N., Phanuphak, N., Grinsztejn, B., Prestage, G. P., Zablotska-Manos, I. B., et al. (2018). Viral suppression and HIV transmission in serodiscordant male couples: an international, prospective, observational, cohort study. Lancet HIV 5, e438-e447. doi: 10.1016/S2352-3018(18)30132-2 van der Sluis et al. describe the potential of using plasmacytoid dendritic cells (pDCs) as an immunotherapy. PDCs are mostly known for their type I interferon-producing capacity, which can enhance NK cell activation and killing. However, pDCs can also improve $\mathrm{T}$ cell immunity by delivering antigens or therapeutic peptides combined with interferons.

Gardner provides an extensive overview of the potential of using recombinant Adeno-Associated Virus Vectors (rAAVs) to deliver bNAbs and engineered HIV inhibitors. Using rAAVs to deliver bNAbs or inhibitors would overcome the need for continued passive administration to sustain expression of these molecules in PLWH.

Finally, Ahlenstiel et al. summarize the recent advances toward developing an alternative HIV cure strategy, termed "block and lock." This approach proposes to permanently silence HIV expression by "blocking" HIV transcription and "locking" the HIV promotor in a permanently latent state. Achievement of this premise would provide a functional cure, where a person still lives with HIV but the presence of the virus is not harmful in the absence of ART.

In summary, the articles presented in this special issue of Frontiers in Cellular and Infection Microbiology give an overview of the obstacles to developing successful HIV cure strategies. Additionally, the articles provide a collection of excellent novel ideas that could advance efforts to reduce the burden of integrated HIV DNA in ART-treated PLWH.

\section{AUTHOR CONTRIBUTIONS}

All authors contributed to the article and approved the submitted version.

\section{FUNDING}

This project has received funding from the European Union's Horizon 2020 research and innovation programme under the Marie Skłodowska-Curie grant agreement No 754513 and The Aarhus University Research Foundation (RMS).

Chew, G. M., Fujita, T., Webb, G. M., Burwitz, B. J., Wu, H. L., Reed, J. S., et al. (2016). TIGIT Marks Exhausted T Cells, Correlates with Disease Progression, and Serves as a Target for Immune Restoration in HIV and SIV Infection. PLoS Pathog. 12, e1005349. doi: 10.1371/journal.ppat.1005349

Chun, T. W., Carruth, L., Finzi, D., Shen, X., Digiuseppe, J. A., Taylor, H., et al. (1997). Quantification of latent tissue reservoirs and total body viral load in HIV-1 infection. Nature 387, 183-188. doi: 10.1038/387183a0

Cohen, M. S., Chen, Y. Q., Mccauley, M., Gamble, T., Hosseinipour, M. C., Kumarasamy, N., et al. (2011). Prevention of HIV-1 infection with early antiretroviral therapy. N. Engl. J. Med. 365, 493-505. doi: 10.1056/NEJMoa1105243

Colby, D. J., Trautmann, L., Pinyakorn, S., Leyre, L., Pagliuzza, A., Kroon, E., et al. (2018). Rapid HIV RNA rebound after antiretroviral treatment interruption in persons durably suppressed in Fiebig I acute HIV infection. Nat. Med. 24, 923 926. doi: 10.1038/s41591-018-0026-6 
Davey, R.T. Jr., Bhat, N., Yoder, C., Chun, T. W., Metcalf, J. A., Dewar, R., et al. (1999). HIV-1 and T cell dynamics after interruption of highly active antiretroviral therapy (HAART) in patients with a history of sustained viral suppression. Proc. Natl. Acad. Sci. U. S. A. 96, 15109-15114. doi: 10.1073/ pnas.96.26.15109

Dufour, C., Gantner, P., Fromentin, R., and Chomont, N. (2020). The multifaceted nature of HIV latency. J. Clin. Invest. 130, 3381-3390. doi: 10.1172/JCI136227

Finzi, D., Hermankova, M., Pierson, T., Carruth, L. M., Buck, C., Chaisson, R. E., et al. (1997). Identification of a reservoir for HIV-1 in patients on highly active antiretroviral therapy. Science 278, 1295-1300. doi: 10.1126/science.278. 5341.1295

Kim, Y., Anderson, J. L., and Lewin, S. R. (2018). Getting the "Kill" into "Shock and Kill": Strategies to Eliminate Latent HIV. Cell Host Microbe 23, 14-26. doi: 10.1016/j.chom.2017.12.004

Pitman, M. C., Lau, J. S. Y., Mcmahon, J. H., and Lewin, S. R. (2018). Barriers and strategies to achieve a cure for HIV. Lancet HIV 5, e317-e328. doi: 10.1016/ S2352-3018(18)30039-0

Rodger, A. J., Cambiano, V., Bruun, T., Vernazza, P., Collins, S., Degen, O., et al. (2019). Risk of HIV transmission through condomless sex in serodifferent gay couples with the HIV-positive partner taking suppressive antiretroviral therapy (PARTNER): final results of a multicentre, prospective, observational study. Lancet 393, 2428-2438. doi: 10.1016/S0140-6736(19)30418-0
Tauriainen, J., Scharf, L., Frederiksen, J., Naji, A., Ljunggren, H. G., Sonnerborg, A., et al. (2017). Perturbed CD8(+) T cell TIGIT/CD226/PVR axis despite early initiation of antiretroviral treatment in HIV infected individuals. Sci. Rep. 7, 40354. doi: 10.1038/srep40354

Volberding, P. A., and Deeks, S. G. (2010). Antiretroviral therapy and management of HIV infection. Lancet 376, 49-62. doi: 10.1016/S0140-6736 (10)60676-9

Wong, J. K., Hezareh, M., Gunthard, H. F., Havlir, D. V., Ignacio, C. C., Spina, C. A., et al. (1997). Recovery of replication-competent HIV despite prolonged suppression of plasma viremia. Science 278, 1291-1295. doi: 10.1126/ science.278.5341.1291

Conflict of Interest: The authors declare that the research was conducted in the absence of any commercial or financial relationships that could be construed as a potential conflict of interest.

Copyright (c) 2021 van der Sluis, Finzi and Parsons. This is an open-access article distributed under the terms of the Creative Commons Attribution License (CC BY). The use, distribution or reproduction in other forums is permitted, provided the original author(s) and the copyright owner(s) are credited and that the original publication in this journal is cited, in accordance with accepted academic practice. No use, distribution or reproduction is permitted which does not comply with these terms. 